\title{
¿Cómo influyen los conocimientos de bioseguridad en las prácticas que realizan los enfermeros limeños?
}

\author{
Yliana Ccarhuarupay-Delgado ${ }^{1}$ \\ Karen Cruzado-Flores ${ }^{2}$
}

RESUMEN: El objetivo fue analizar la fuerza de asociación entre los niveles del conocimiento y prácticas de bioseguridad en los licenciados de enfermería. La muestra fue de 185 licenciados de enfermería de dos hospitales nacionales de Lima. Las variables del estudio fueron: conocimientos y prácticas sobre la bioseguridad. Se empleó la bondad de ajuste de la prueba Hosmer-Lemeshow. De los 185 enfermeros un 50.4\% tenía un conocimiento regular de bioseguridad y un $70.8 \%$ de los mismos realizaban una práctica apropiada de bioseguridad. Se observa una mayor fuerza de asociación entre el conocimiento bueno y la práctica apropiada de bioseguridad de los enfermeros en comparación con los que tienen un conocimiento regular (OR:2.44). Respecto al conocimiento bueno de la dimensión de medidas de bioseguridad se asoció con una práctica apropiada de bioseguridad (OR:4.38). El conocimiento bueno de bioseguridad influye en la práctica apropiada de la misma en comparación con un conocimiento regular. Se promueve la elaboración de guías de procedimientos acerca de las medidas de bioseguridad según las características del área laboral. Asimismo se recomienda incentivar en los establecimientos de salud el uso de materiales de protección con el fin de aplicar las medidas de bioseguridad de manera eficaz.

PALABRAS CLAVE: Exposición a Agentes Biológicos; Nivel de conocimiento; Medidas de seguridad; Licenciados.

Citar como: Ccarhuarupay Y, Cruzado K. ¿Cómo influyen los conocimientos de bioseguridad en las prácticas que realizan los enfermeros limeños? CASUS. 2017;2(1):54-61.

\footnotetext{
${ }^{1}$ Hospital Cayetano Heredia. Licenciada en enfermería. ${ }^{2}$ Hospital Nacional Hipólito Unanue. Licenciada en enfermería.

Correo electrónico: yliccd1@gmail.com

Recibido: 18-01-2017 Aprobado: 01-04-2017
} 


\section{INTRODUCCIÓN}

Debido a los riesgos biológicos y a la aparición de enfermedades contagiosas con potencial epidémico que afecta a la salud ocupacional, diversas organizaciones consideran que la bioseguridad es importante para la gestión de calidad ante eventos accidentales o intencionales. Esto puede ser a través de la introducción de las medidas preventivas en diferentes establecimientos de salud y laboratorios en los que hay mayor riesgo biológico tanto para el personal de salud como para el paciente (1).

La Organización Internacional del Trabajo (OIT) informó que en el 2015 cada 15 segundos un trabajador muere a causa de accidentes o enfermedades relacionadas con el trabajo. Cada día mueren 6300 personas a causa de accidentes o enfermedades relacionadas con el trabajo y ocurren más de 2.3 millones de muertes por año. Anualmente sobrevienen más de 317 millones de accidentes en el trabajo que ocasionan el ausentismo laboral. Del mismo modo esto produce un costo tanto para el hospital como para el trabajador (2).

Las principales causas de estos accidentes laborales que se dan principalmente en el personal de salud son los riesgos biológicos. Como consecuencia los trabajadores pueden sufrir diversas enfermedades infecciosas siendo las más frecuentes los accidentes de tipo punzo cortante y enfermedades transmisibles como la hepatitis por virus B (HvB) y C, el VIH y el tétano (3-5). Es por ello que el Ministerio de Salud (MINSA) creó el Manual de Salud Ocupacional el cual está dirigido a la promoción y protección de la salud de los trabajadores, la prevención de accidentes de trabajo y enfermedades ocupacionales causadas por las condiciones de trabajo. Este manual está regido bajo tres principios básicos: universalidad, uso de barreras y medios de eliminación de material contaminado (6).

Los enfermeros asistenciales que laboran en hospitales u otros establecimientos de salud están potencialmente expuestos a los riesgos biológicos (7). Además se tiene que tener en cuenta las enfermedades que los pacientes puedan transmitir, ya que en su mayoría ponen en constante riesgo a los enfermeros que pasan la mayor parte del tiempo a disposición del enfermo en su cuidado diario. Por esta razón es importante que los enfermeros tengan conocimientos sobre las medidas de bioseguridad que posteriormente deben aplicar en sus diversos procedimientos para aminorar aquellas situaciones que ponen en peligro su salud y la de los pacientes. Ya que por su propia patología el paciente es vulnerable a adquirir complicaciones que puedan agravar su estado de salud (8).

Estudios evidencian que los enfermeros asistenciales que tenían un conocimiento alto de bioseguridad tenían una práctica apropiada de la misma. Asimismo los que tenían conocimientos regulares de bioseguridad brindaban una práctica medianamente apropiada en los diversos procedimientos invasivos $(8-10)$.

Debido a esta problemática y los pocos estudios a nivel nacional que indagan en la asociación entre los niveles de conocimiento y prácticas de bioseguridad el presente estudio tuvo como objetivo analizar la fuerza de asociación entre los niveles de conocimientos y prácticas de la bioseguridad en los licenciados de enfermería en dos hospitales nacionales de Lima.

\section{MATERIALES Y MÉTODOS}

El estudio fue transversal descriptivo, correlacional y explicativo. La muestra fue de 185 licenciados de enfermería de dos hospitales nacionales de Lima. De los cuales 99 eran licenciados de un hospital nacional del Lima Norte y 86 de un hospital nacional de Lima Este. El muestreo fue no probabilístico. Se incluyeron a los enfermeros asistenciales de ambos sexos entre 25 y 55 años de edad, que tenían más de seis meses trabajando en la misma institución y en diferentes servicios que incluyeran adultos hospitalizados. Se excluyeron a los enfermeros con carga administrativa.

Para medir la variable dependiente práctica sobre la bioseguridad se empleó un cuestionario de autoevaluación de 16 preguntas acerca del cumplimiento de las normas de bioseguridad, lavado de manos, uso de barreras (guantes, tapabocas, bata, protección ocular y gorro), eliminación correcta de residuos, preocupaciones ante la exposición de un riesgo biológico, entre otros (11). La práctica fue categorizada en: medianamente apropiada y apropiada. La misma fue validada mediante jueces expertos con un alfa de Cronbach de 0.69 .

La variable conocimiento sobre bioseguridad fue medida mediante un cuestionario de 19 preguntas clasificadas en tres dimensiones: agentes biológicos, medidas de bioseguridad y manejo de residuos 
sólidos. La misma fue categorizada en conocimiento deficiente, regular y bueno (12). El cuestionario fue validado mediante jueces expertos con un alfa de Cronbach de 0.70 .

Las variables sociodemográficas fueron: edad, variable categórica (25-35; 36-45 y 46-55 años); sexo, variable categórica (femenino y masculino); estado civil, variable categórica (soltero, casado y conviviente); antigüedad laboral, variable categórica (6 meses a 5 años, 6 años a 15 años y 16 a más años); docente, variable categórica (sí y no); grado académico alcanzado, variable categórica (licenciatura, especialidad y maestría); modalidad de contrato, variable categórica (nombrado, CAS y terceros); estado vacunal, definida como la aplicación de las vacunas HvB y la difteria tétano (DT). La misma se categorizó en completo e incompleto.

Se utilizó el programa STATA 12 para la realización del análisis estadístico. Se calculó frecuencias y porcentajes para las variables categóricas. Respecto al análisis bivariado se utilizó la prueba estadística
Chi-cuadrado. Asimismo, se realizó la bondad de ajuste del modelo con la prueba de Hosmer-Lemeshow. Posteriormente se empleó el modelo de regresión logística ordinal, donde se calculó Odds Ratio (OR) e intervalos de confianza con una significancia estadística menor o igual a 0.05 .

Para recolectar los datos el estudio contó con la aprobación del comité de ética institucional. Asimismo se respetó la confidencialidad mediante el anonimato de los encuestados. Cabe resaltar que no hubo riesgos para la salud de los mismos debido a las características del estudio.

\section{RESULTADOS}

En la tabla 1 se muestra que de los 185 encuestados el $40 \%$ tenía entre 36 y 45 años. La mayoría fueron mujeres $(83.8 \%)$, nombrados $(66.5 \%)$, no docentes $(90.8 \%)$ y cuentan con licenciatura $(55.7 \%)$. Más de la mitad pertenecían al hospital de Lima Norte. Por último un $50.4 \%$ de los encuestados tenía un conocimiento regular de bioseguridad y un $70.8 \%$ de los mismos realizaban una práctica apropiada de bioseguridad.

\section{Tabla 1. Descripción de la características de los enfermeros}

\begin{tabular}{|c|c|c|}
\hline & $\mathrm{n}$ & $(\%)$ \\
\hline \multicolumn{3}{|l|}{ Edad } \\
\hline 25-35 años & 81 & 43.8 \\
\hline 36-45 años & 74 & 40.0 \\
\hline 46-55 años & 30 & 16.2 \\
\hline \multicolumn{3}{|l|}{ Sexo } \\
\hline Mujer & 155 & 83.8 \\
\hline Hombre & 30 & 16.2 \\
\hline \multicolumn{3}{|l|}{ Estado civil } \\
\hline Casado & 90 & 48.7 \\
\hline Soltero & 80 & 43.2 \\
\hline Conviviente & 15 & 8.1 \\
\hline \multicolumn{3}{|c|}{ Antigüedad laboral } \\
\hline 6 meses - 5 años & 78 & 42.2 \\
\hline 6 años - 15 años & 96 & 51.9 \\
\hline 16 años a más & 11 & 5.9 \\
\hline \multicolumn{3}{|l|}{ Docente } \\
\hline No & 168 & 90.8 \\
\hline Sí & 17 & 9.2 \\
\hline
\end{tabular}


Máximo grado académico alcanzado

Licenciatura

Especialidad

Maestría

Modalidad de contrato

Nombrado

CAS

Tercero

Estado vacunal

Completo

Incompleto

Hospital

Lima Este

Lima Norte

Conocimiento sobre la bioseguridad

Regular

Bueno

Agentes biológicos

Bueno

Regular

Medidas de bioseguridad

Bueno

Regular

Manejo de residuos hospitalarios

Bueno

Prácticas de bioseguridad

Medianamente apropiada

Tener un conocimiento regular se relacionó con una práctica medianamente apropiada de bioseguridad en los enfermeros (37.9\%). Mientras que un conocimiento bueno se asoció con una práctica apropiada de bioseguridad $(80.0 \%)$. La dimensión medidas de bioseguridad se relacionó con una práctica apropiada de bioseguridad. Las demás dimensiones y las variables sociodemográficas relacionadas a las prácticas de bioseguridad no mostraron significancia (ver tabla 2). 
Tabla 2. Asociación descriptiva entre el conocimiento y prácticas de bioseguridad

\begin{tabular}{|c|c|c|c|}
\hline & Nivel $d$ & ctica & \\
\hline & $\begin{array}{c}\text { Medianamente } \\
\mathrm{n}(\%)\end{array}$ & $\begin{array}{c}\text { Apropiada } \\
\mathrm{n}(\%)\end{array}$ & p-value \\
\hline $\begin{array}{l}\text { Conocimiento sobre la bioseguridad } \\
\text { Regular } \\
\text { Bueno }\end{array}$ & $\begin{array}{l}36(37.9) \\
18(20.0)\end{array}$ & $\begin{array}{l}59(62.1) \\
72(80.0)\end{array}$ & 0.00 \\
\hline $\begin{array}{l}\text { Agentes biológicos } \\
\text { Regular } \\
\text { Bueno }\end{array}$ & $\begin{array}{c}6(46.2) \\
48(27.9)\end{array}$ & $\begin{array}{c}57(53.9) \\
124(72.1)\end{array}$ & 0.16 \\
\hline $\begin{array}{l}\text { Medidas de bioseguridad } \\
\text { Regular } \\
\text { Bueno }\end{array}$ & $\begin{array}{c}8(61.5) \\
46(26.7)\end{array}$ & $\begin{array}{c}5(38.5) \\
126(73.3)\end{array}$ & 0.00 \\
\hline $\begin{array}{l}\text { Manejo de residuos hospitalarios } \\
\text { Regular } \\
\text { Bueno }\end{array}$ & $\begin{array}{c}8(40.0) \\
46(27.9)\end{array}$ & $\begin{array}{c}12(60.0) \\
119(72.1)\end{array}$ & 0.26 \\
\hline $\begin{array}{l}\text { Edad } \\
25-35 \text { años } \\
36-45 \text { años } \\
46-55 \text { años }\end{array}$ & $\begin{array}{l}35(43.2) \\
35(47.3) \\
17(56.7)\end{array}$ & $\begin{array}{l}46(56.8) \\
39(52.7) \\
13(43.3)\end{array}$ & 0.45 \\
\hline $\begin{array}{l}\text { Sexo } \\
\text { Mujer } \\
\text { Hombre }\end{array}$ & $\begin{array}{l}74(47.7) \\
13(43.3)\end{array}$ & $\begin{array}{l}81(52.3) \\
17(56.7)\end{array}$ & 0.66 \\
\hline $\begin{array}{l}\text { Estado civil } \\
\text { Casado } \\
\text { Soltero } \\
\text { Conviviente }\end{array}$ & $\begin{array}{l}43(47.8) \\
34(42.5) \\
10(66.7)\end{array}$ & $\begin{array}{c}47(52.2) \\
46(57.5) \\
5(33.3)\end{array}$ & 0.22 \\
\hline $\begin{array}{l}\text { Antigüedad laboral } \\
6 \text { meses }-5 \text { años } \\
6 \text { años }-15 \text { años } \\
16 \text { años a más }\end{array}$ & $\begin{array}{l}33(42.3) \\
47(48.9) \\
7(63.6)\end{array}$ & $\begin{array}{c}45(57.7) \\
49(51.0) \\
4(36.4)\end{array}$ & 0.35 \\
\hline $\begin{array}{l}\text { Docente } \\
\text { No } \\
\text { Sí }\end{array}$ & $\begin{array}{c}79(47.0) \\
8(47.1)\end{array}$ & $\begin{array}{c}89(52.9) \\
9(52.9)\end{array}$ & 0.99 \\
\hline $\begin{array}{l}\text { Máximo grado académico alcanzado } \\
\text { Licenciatura } \\
\text { Especialidad } \\
\text { Maestría }\end{array}$ & $\begin{array}{l}51(49.5) \\
33(45.2) \\
3(33.3)\end{array}$ & $\begin{array}{l}52(50.5) \\
40(54.8) \\
6(66.7)\end{array}$ & 0.59 \\
\hline $\begin{array}{l}\text { Modalidad de contrato } \\
\text { Nombrado } \\
\text { CAS } \\
\text { Tercero }\end{array}$ & $\begin{array}{l}59(47.9) \\
16(45.7) \\
12(44.4)\end{array}$ & $\begin{array}{l}64(52.0) \\
19(54.3) \\
15(55.6)\end{array}$ & 0.93 \\
\hline $\begin{array}{l}\text { Estado vacunal } \\
\text { Completo } \\
\text { Incompleto }\end{array}$ & $\begin{array}{l}74(45.9) \\
13(54.1)\end{array}$ & $\begin{array}{l}87(54.0) \\
11(45.8)\end{array}$ & 0.45 \\
\hline $\begin{array}{l}\text { Hospital } \\
\text { Lima Este } \\
\text { Lima Norte }\end{array}$ & $\begin{array}{l}40(46.5) \\
47(47.5)\end{array}$ & $\begin{array}{l}46(53.5) \\
52(52.5)\end{array}$ & 0.89 \\
\hline
\end{tabular}


En la tabla 3 se observa una mayor fuerza de asociación entre el conocimiento bueno y la práctica apropiada de bioseguridad de los enfermeros en comparación con los que tienen un conocimiento regular (OR: 2.44; IC 95\%: 1.26-4.73). Asimismo un conocimiento bueno respecto a las medidas de bioseguridad se asoció con una práctica apropiada de bioseguridad en los enfermeros (OR: 4.38; IC 95\%: 1.36-14.18). Las demás dimensiones no fueron significativas en la asociación. La bondad de ajuste del modelo fue de 0.08 .

Tabla 3 Fuerza de asociación entre los conocimientos y las prácticas de bioseguridad

\begin{tabular}{lccc}
\hline & \multicolumn{2}{c}{ Nivel de práctica } & \\
& OR & IC 95\% & p-value \\
\hline $\begin{array}{l}\text { Conocimiento sobre la bioseguridad } \\
\text { Regular } \\
\text { Bueno }\end{array}$ & 1.00 & & \\
$\begin{array}{l}\text { Agentes biológicos } \\
\text { Regular } \\
\text { Bueno }\end{array}$ & 2.44 & - & - \\
Medidas de bioseguridad & & & 0.00 \\
$\begin{array}{l}\text { Regular } \\
\text { Bueno }\end{array}$ & 1.00 & - & - \\
Manejo de residuos hospitalarios & 2.21 & $0.71-6.92$ & 0.17 \\
Regular & & & \\
Bueno & 1.00 & - & - \\
& 4.38 & $1.36-14.18$ & 0.01 \\
\hline
\end{tabular}

\section{DISCUSIÓN}

En el estudio el $50.4 \%$ de los encuestados tenía un conocimiento regular de bioseguridad y un $70.8 \%$ realizaban una práctica apropiada de bioseguridad. Además se observó una mayor fuerza de asociación entre el conocimiento bueno y la práctica apropiada de bioseguridad de los enfermeros en comparación con los que tienen un conocimiento regular. Asimismo un conocimiento bueno sobre las medidas de bioseguridad se asoció positivamente con una práctica apropiada de bioseguridad en los enfermeros.

Con un aumento del conocimiento de bioseguridad la práctica apropiada aumenta de manera proporcional en los enfermeros. Este hallazgo es similar en otros estudios $(8,10,13)$. Los enfermeros asistenciales cumplen con diversos procedimientos invasivos en su área laboral. Por esta razón tienen que tener un conocimiento previo de bioseguridad para la realización de los mismos. El conocimiento es importante porque permite identificar diversos riesgos tanto para el profesional como para los pacientes. Con el fin de prevenir complicaciones sobre todo en la manipulación de objetos punzo cortantes (14). Por esta razón los conocimientos de bioseguridad, la ética profesional y los derechos de los pacientes exigen a los licenciados de enfermería intervenir eficazmente en los procedimientos con el fin de prevenir la aparición de enfermedades o muerte de los pacientes (15-18).

Por otro lado, el presente estudio evidenció que la dimensión medidas de bioseguridad se asoció con una práctica apropiada $(8,10)$. Cabe resaltar que está dimensión comprende el lavado de manos y el uso de barreras actividades que son habituales en el campo laboral del enfermero (8). Los enfermeros debido a la importancia de estas medidas así como a la frecuencia con que las realizan tienden a tener un conocimiento mayor sobre las mismas (8).

Entre las limitaciones cabe señalar que aunque el instrumento empleado evalúa correctamente la práctica apropiada se debería tomar en cuenta la observación de los procedimientos que hace el enfermero para una mayor precisión. Respecto al diseño del estudio se señala el sesgo de memoria y de selección. Se recomienda realizar estudios en muestras más amplias que comparen hospitales públicos y privados. Asimismo realizar estudios experimentales donde se evalúen la eficacia de intervenciones enfocadas en la prevención y enseñanza de la bioseguridad tanto en los profesionales como en los pacientes. 


\section{CONCLUSIONES}

Un nivel bueno de conocimientos influye positivamente en la práctica apropiada de bioseguridad. Asimismo un nivel de conocimiento bueno en la dimensión de medidas de bioseguridad se asocia positivamente con la práctica apropiada de la misma. Se debe promover la elaboración de guías de procedimientos acerca de las medidas de bioseguridad según las características del área laboral. Asimismo se incentiva que los enfermeros se capaciten para actualizar sus conocimientos. Por otro lado se recomienda incentivar en los establecimientos de salud el uso de materiales de protección con el fin de aplicar las medidas de bioseguridad de manera eficaz.

\section{REFERENCIAS BIBLIOGRÁFICAS}

1. Organización Panamericana de la Salud. Bioseguridad y mantenimiento [Internet].

Washington, EEUU: OPS; 2005 [citado el 02 de marzo del 2017]. Disponible en:

http://www2.paho.org/hq/index.php? option $=$ com content $\&$ view $=$ article $\&$ $\mathrm{id}=5460 \% 3 \overline{\mathrm{A}} 2011$-bioseguridad-ma ntenimiento\&catid $=3612 \% 3$ Alaborat ory-services-contents $\&$ Itemid $=3952$ \&lang $=\mathrm{es}$

2. Organización Internacional del Trabajo. Seguridad y salud en el trabajo [Internet]. Ginebra, Suezia: OIT; 2017 [citado el 15 de marzo del 2017]. Disponible en:

http://ilo.org/global/topics/safety-and -health-at-work/lang--es/index.htm

3. Tomasina F, Gómez F. Accidentes laborales en el Hospital de Clínicas. Rev Med Uruguay 2001; 17(3):156-160.

4. Bonagamba $\mathrm{M}$, Palucci $\mathrm{M}$, et al. Accidentes de Trabajo con Material Biológico entre Trabajadores de Unidades de Salud Pública. Rev Latino-am Enfermagem 2007; 15(4).

5. Benavides F, Delclos $\mathrm{J}$, et al. Lesiones por accidentes de trabajo, una prioridad en salud pública. Rev. Esp. Salud Pública Madrid 2006; 80(5):553-565.

6. Ministerio de Salud. Manual de Salud Ocupacional [Internet]. Lima, Perú: MINSA; 2005 [citado el 19 de marzo del 2017]. Disponible en: http://www.digesa.minsa.gob.pe/pub licaciones/descargas/manual deso.P DF

7. Romero, A. Personal de Enfermería: condiciones de trabajo de alto riesgo. Salud de los Trabajadores, 1998; 6(2):113-119.
8. Liberato J. Relación entre nivel de conocimiento y cumplimiento de la práctica de medidas de bioseguridad del Profesional de Enfermería en el Centro Quirúrgico del Instituto Nacional de Oftalmología INO - 2009 [Tesis de Especialidad]. Lima: Universidad Nacional Mayor de San Marcos; 2013.

9. Huaman D, Romero L. Nivel de conocimiento y practica de medidas de bioseguridad en las enfermeras de los servicios de medicina del Hospital Belén de Trujillo 2014 [Tesis de Licenciatura]. Lima: Universidad Privada Antenor Orrego; 2014.

10. Mestanza M. Relación entre conocimientos y prácticas sobre las medidas de bioseguridad en el profesional de enfermería de sala de operaciones del Hospital Nacional Hipólito Unanue 2008 [Tesis de Especialidad]. Lima: Universidad Nacional Mayor de San Marcos; 2009.

11. Díaz A. Conocimiento en Riesgos Biológicos y Prácticas de Bioseguridad en el Personal Docente de la Facultad de Salud de una Institución de Educación Superior de la Ciudad de Cali [Tesis de Maestría]. Santiago de Cali: Universidad del Valle; 2013.

12. Alarcón $\mathrm{M}$, Rubiños $\mathrm{S}$. Conocimientos y Prácticas en la Prevención de Riesgos Biológicos de las Enfermeras del Hospital Belen Lambayeque, 2012 [Tesis de Licenciatura]. Lima: Universidad Católica Santo Toribio de Mogrovejo; 2013.

13. Chávez Q, Reyna R. Relación entre conocimientos y actitudes del profesional de enfermería hacia la aplicación de las medidas de bioseguridad en la Unidad Crítica en el Hospital Nacional Hipólito Unanue. Lima 2013 [Tesis Doctoral]. Lima:
Universidad Nacional Mayor de San Marcos; 2013.

14. Hernández E, Acosta $\mathrm{M}$, et al. Intervención educativa para incrementar los conocimientos sobre bioseguridad en el personal de enfermería de una institución hospitalaria. Rev Cubana Enfermer 2006; 22(2).

15. Téllez J, Tovar M. Medidas de Bioseguridad que Aplica el Profesional de Enfermería y la Accidentabilidad Laboral en la Unidad Quirúrgica, Hospital "Dr. José María Vargas" en el Segundo Semestre de 2007 [Tesis de Licenciatura]. Caracas: Universidad Central de Venezuela Facultad de Medicina Escuela de Enfermería; 2008.

16. El Peruano. Normas Legales [Internet]. Lima, Perú: El Peruano; 2005 [citado el 25 de marzo del 2017]. Disponible en: http://busquedas.elperuano.com.pe/d ownload/url/decreto-supremo-que-a prueba-el-reglamento-del-decreto-le gisl-decreto-supremo-n-002-2017-ju s-1479011-1

17. Trincado M, Ramos I, et al. Evaluación de las normas de bioseguridad en el servicio de hemodiálisis del Instituto de Nefrología "Dr. Abelardo Buch López", 2009. Rev Cubana Hig Epidemiol. 2011: 49(3):356-372.

18. Chávez V. Relación entre el nivel de conocimientos sobre medidas de bioseguridad y su aplicación por los profesionales de enfermería que laboran en los servicios de Centro Quirúrgico y Hospitalización del Instituto Regional de Enfermedades Neoplásicas Iren Norte [Tesis de Especialidad]. Lima: Universidad Nacional Mayor de San Marcos; 2012. 


\section{How do biosafety knowledge influence practices carried out by Lima nurses?}

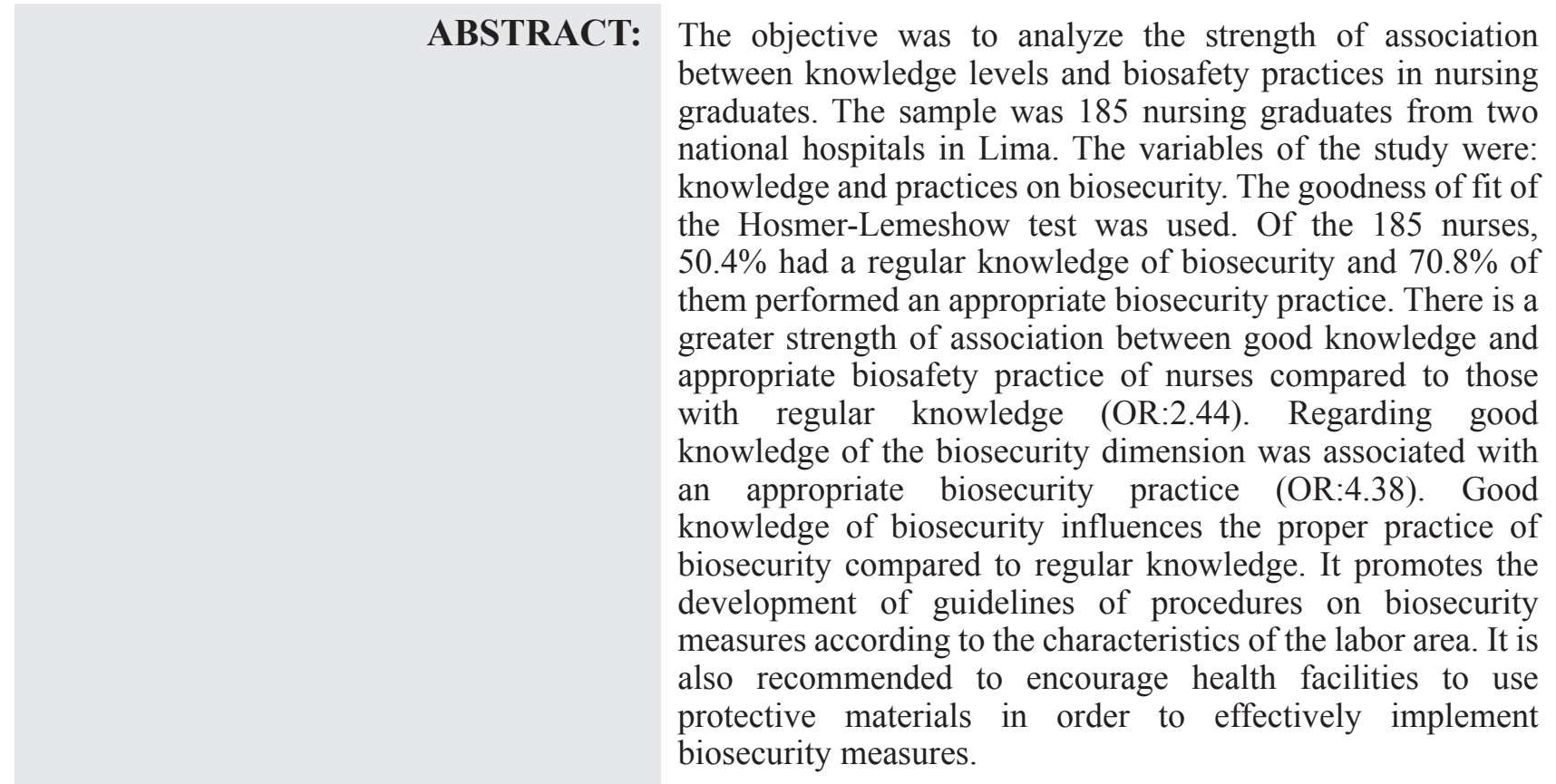

KEY WORDS: Exposure to Biological Agents; Level of knowledge; security measures; Nursing. 